\title{
Orthostatic Headache in Children Including Postural Tachycardia Syndrome and Orthostatic Hypotension: A Near-Infrared Spectroscopy Study
}

\author{
Soken Go ${ }^{1,2, *(D)}$, Gaku Yamanaka ${ }^{1}$, Akiko Kasuga ${ }^{1}$, Kanako Kanou ${ }^{1}$, Tomoko Takamatsu ${ }^{1}$, \\ Mika Takeshita ${ }^{1}$, Natsumi Morishita ${ }^{1}$, Shinichiro Morichi ${ }^{1}{ }^{1},{ }$, Yu Ishida ${ }^{1}$, Shingo Oana ${ }^{1}$, \\ Yasuyo Kashiwagi ${ }^{1}$, Takashi Mitsufuji ${ }^{3}$, Nobuo Araki ${ }^{3}$ and Hisashi Kawashima ${ }^{1}$ \\ 1 Department of Pediatrics and Adolescent Medicine, Tokyo Medical University, Tokyo 160-0023, Japan; \\ gaku19710911@gmail.com (G.Y.); tea-party.alice@hotmail.co.jp (A.K.); \\ kanako.hayashi.0110@gmail.com (K.K.); twomoonchild@gmail.com (T.T.); jerryfish_mika@yahoo.co.jp (M.T.); \\ sunflowernk69@gmail.com (N.M.); shinichiro-m@hotmail.co.jp (S.M.); y.ishida31@gmail.com (Y.I.); \\ shingooana@yahoo.co.jp (S.O.); hoyohoyo18@hotmail.com (Y.K.); hisashi@tokyo-med.ac.jp (H.K.) \\ 2 Department of Psychosomatic Medicine, Tokyo Metropolitan Children's Medical Center, \\ Tokyo 183-8561, Japan \\ 3 Department of Neurology, Saitama Medical University, Saitama 350-0495, Japan; \\ mitsufuj@saitama-med.ac.jp (T.M.); arakin@saitama-med.ac.jp (N.A.) \\ * Correspondence: soupei@tokyo-med.ac.jp; Tel.: +81-3-3342-6111; Fax: +81-3-3344-0643
}

Received: 30 November 2020; Accepted: 21 December 2020; Published: 21 December 2020

\begin{abstract}
Background and aim: Although head and/or neck pain attributed to orthostatic hypotension is included in international guidelines, its mechanisms and relevance remain unknown. This study examined the term's relevance and aimed to elucidate the associated clinical features. Methods: An active stand test was performed to evaluate fluctuations in systemic and cerebral circulation in children and adolescents reporting complaints in the absence of a confirmed organic disorder The subjects were categorized based on orthostatic headache presence/absence, and their characteristics and test results were compared. Results: Postural tachycardia syndrome was observed in $50.0 \%$ of children with, and $55.1 \%$ without, orthostatic headache. For orthostatic hypotension, the respective values were $31.3 \%$ and $30.6 \%$. A history of migraine was more prevalent in children with orthostatic headaches $(64.1 \%$ vs. $28.6 \% ; p<0.01)$. The observed decrease in the cerebral oxygenated hemoglobin level was larger in children with orthostatic headaches (Left: 6.3 (3.2-9.4) vs. $4.1(0.8-6.1) ; p<0.01$, Right: 5.3 (3.1-8.6) vs. $4.0(0.8-5.9) ; p<0.01)$. Conclusion: Fluctuations in cerebral blood flow were associated with orthostatic headaches in children, suggesting that the headaches are due to impaired intracranial homeostasis. As orthostatic headache can have multiple causes, the term "head and/or neck pain attributed to orthostatic (postural) hypotension" should be replaced with a more inclusive term.
\end{abstract}

Keywords: head and/or neck pain; the International Classification of Headache Disorders 3rd edition; children; cerebral blood flow fluctuation; migraine; postural tachycardia syndrome; pediatric; adolescent; headache; orthostatic dysregulation; orthostatic intolerance; orthostatic headache; cerebrospinal fluid leak; active standing test; head up-tilt

\section{Introduction}

Headaches are common in childhood and adolescence, with approximately $20 \%$ of children reporting having a headache at least once a year [1]. In children, recurrent headaches are predominantly primary headaches, such as migraine or tension-type headaches. 
Chronic headaches may greatly interfere with daily life. Children with orthostatic headaches experience difficulties in daily life. However, their condition is difficult to treat because, being induced by postural changes such as standing, it does not meet the diagnostic criteria for either primary or secondary headaches. When moving from the supine to a standing position, the volume of blood that returns to the heart decreases by approximately $30 \%$, lowering cardiac output and systemic blood pressure and triggering the baroreceptor reflex. Although this reflex maintains adequate, blood pressure in healthy people, those with an abnormal baroreceptor reflex or decreased plasma volume experience a severe drop in blood pressure upon standing. In adults, orthostatic hypotension $(\mathrm{OH})$ is diagnosed when a decrease in systolic blood pressure $\geq 20 \mathrm{mmHg}$ or diastolic blood pressure $\geq 10 \mathrm{mmHg}$ occurs within $3 \mathrm{~min}$ of changing from the supine or sitting to the standing position.

Low et al. published a study of postural tachycardia syndrome (POTS) in 1995 [2]. POTS commonly effects women in their 40s, and many patients first notice their symptoms in childhood or adolescence [3]. The current global diagnostic criteria for POTS in children and adolescents include marked tachycardia $(>40 \mathrm{bpm})$ triggered by $10 \mathrm{~min}$ of active standing after rising from a supine position or a head-up tilt test. The criterion for POTS is a lack of a marked decrease in blood pressure. However, like OH, POTS results from abnormal changes in blood circulation mediated by the autonomic nervous system when assuming a standing position. Thus, POTS is often considered to be similar to $\mathrm{OH}$. As a result that POTS involves orthostatic tachycardia associated with orthostatic symptoms, it is thought to encompass multiple pathological conditions with such symptoms as severe and/or long-lasting fatigue, light-headedness with prolonged sitting or standing, brain fog, forceful heartbeats or palpitations, nausea, abdominal pain, and headache.

A disorder termed "head and/or neck pain attributed to orthostatic (postural) hypotension" was included in the third edition of the International Classification of Headache Disorders (ICHD3). This concept, which has yet to become established, is defined as "pain, mostly in the back of the neck but sometimes spreading upwards to the occipital region ("coat hanger" distribution), attributed to postural hypotension presenting only in upright posture" (ICHD3, A 10.7) [4]. Impaired intracranial homeostasis has been suggested as a possible cause. However, this concept is derived from studies on $\mathrm{OH}$ due to neurological diseases in the elderly, such as Parkinson's disease, multisystem atrophy, and trauma $[5,6]$, and it is unclear whether it applies to orthostatic headaches in children and adolescents. Moreover, it is still unclear whether POTS without $\mathrm{OH}$ is consistent with this disease concept.

The present study examined the relevance of the term, "head and/or neck pain attributed to orthostatic (postural) hypertension," as included in the ICHD3 by investigating the mechanisms of orthostatic headaches in children based on clinical features and cerebral blood flow fluctuations.

\section{Methods}

\subsection{Participants}

Pediatric patients who visited our hospital between January 2017 and March 2019 due to three or more concurrent complaints disrupting daily life and school attendance and lasting more than two months, such as headache, malaise, palpitations, morning fatigue, daily nausea, face pallor, umbilical colic, and orthostatic dizziness, were identified. Patients with an obvious, posttraumatic headache were excluded at the initial visit. The eligible patients underwent an examination which included detailed history taking, neurophysical tests, thoracoabdominal radiography, head magnetic resonance imaging (MRI), echocardiography, 12-lead electrocardiography, and blood analysis, including thyroid hormone measurement. If no clearly organic disorder was found, the subjects and their parents were asked to submit their verbal and written informed consent to participate. All participating subjects underwent the active stand test (described below), and their cerebral blood flow was simultaneously assessed. None of the subjects received any drug affecting their blood pressure or modifying their NE level, such as selective serotonin reuptake inhibitor (SSRI) or atomoxetine, before the test. The participants were divided into those who either did or did not experience a headache induced by the active stand 
test (the orthostatic headache vs. non-orthostatic headache groups). The groups were compared in terms of their characteristics, background factors, and systemic and cerebral circulatory responses. In addition, the association between the presence/absence of pulsatility in induced headaches and headache sites and other factors were examined.

\subsection{Patient Characteristics and Background}

The patient characteristics of age, sex, height, weight, body mass index (BMI), and cardiothoracic ratio (CTR) were examined. The background factors assessed included the presence/absence of migraine and morning fatigue. Migraine was diagnosed based on the ICHD3 criteria.

\subsection{Active Stand Test}

The subjects were admitted on the day before the active stand test. They underwent the test before breakfast on the following day between 8:00 and 9:30 a.m. in a quiet room with an ambient temperature between 23-24 degrees Celsius and no external light. After resting in the supine position for $10 \mathrm{~min}$, they were asked to stand up in $3 \mathrm{~s}$ and remain standing for $10 \mathrm{~min}$. Subsequently, they were asked to return to the supine position and rest for $5 \mathrm{~min}$. Readings were taken continuously throughout this procedure. Blood pressure, pulse, and blood pressure recovery time were measured using a continuous noninvasive pressure system (Finometer MIDI, Finapres Medical Systems) with a small to medium-sized probe placed on the left middle finger (or another finger, depending on the size of their fingers). To determine the timing of subjective symptom onset, the subjects were asked by the examiner whether they had a headache before standing and at every minute until the conclusion of the test. None of the subjects had a headache while in the supine position. Their responses were recorded. After the conclusion of the test, the subjects were asked about the site and features of their headache, if any, and the presence or absence of pulsatility. If the subject felt severe orthostatic intolerance or presyncope symptoms, the test was terminated, and the point was recorded, but there were only few such instances. In addition, to measure the blood norepinephrine (NE) level, an indwelling needle was inserted and fixed to the left cubital vein at least $30 \mathrm{~min}$ before the start of the active stand test. Flashback blood samples were collected before, and $10 \mathrm{~min}$ after, standing. POTS was diagnosed if a sustained heart rate increase of $40 \mathrm{bpm}$ within $10 \mathrm{~min}$ of standing was recorded in the absence of $\mathrm{OH}$.

\subsection{Evaluation of Cerebral Blood Flow}

The probes of an infrared oximeter (NIRO-200 NX, Hamamatsu Photonics) were attached to the left and right sides of the frontal region, and a hairband was used to fix the probes and prevent light leakage. Highly permeable light at 700 to $900 \mathrm{~nm}$ was used, and the light scattered in the body was automatically measured with a detector. The oxygenated hemoglobin $(\mathrm{OxyHb})$ concentrations in the bilateral frontal cerebral cortices were measured using the Modified Beer-Lambert method [7]. To evaluate the change in $\mathrm{OxyHb}$ concentration from immediately before to after standing, the $\mathrm{OxyHb}$ level at the time of headache onset in the orthostatic headache group and the lowest $\mathrm{OxyHb}$ level while standing in the non-orthostatic headache group were analyzed. Determining if there was any correlation between the $\mathrm{OxyHb}$ and clinical symptoms was impossible because the blood pressure recovery time immediately after standing was very brief. Thus, the $\mathrm{OxyHb}$ levels obtained during the recovery time were not included in the analysis.

\subsection{Statistical Analysis}

SPSS 25.0 (SPSS Inc., Chicago, IL, USA) was used to perform the Mann-Whitney and chi-squared tests. $p<0.01$ was considered to indicate statistical significance. 


\subsection{Ethical Approval and Details of Study Protocol Registration}

Written informed consent was obtained from all the subjects for the publication of the details of their case. A copy of their written consent is available for review upon request. All procedures involving human participants were done in accordance with the ethical standards of the institutional and national research committees (Ethics Committee of the Tokyo Medical University; SH3337) and the 1964 Helsinki Declaration and its later amendments or comparable ethical standards. As this study is a clinical audit of ICHD3 criteria, it does not necessarily require ethics approval.

\section{Results}

The inclusion criteria were met by 113 subjects who subsequently underwent the active stand test, including 64 in the orthostatic headache group and 49 in the non-orthostatic headache group (Figure 1). POTS was found in 32 subjects (50.0\%) in the orthostatic headache group, and 27 subjects $(55.1 \%)$ in the non-orthostatic headache group. $\mathrm{OH}$ was found in $20(31.3 \%)$ and $15(30.6 \%)$ subjects in the orthostatic headache and non-orthostatic headache groups, respectively. No statistically significant difference was observed between groups $(p=0.876)$.

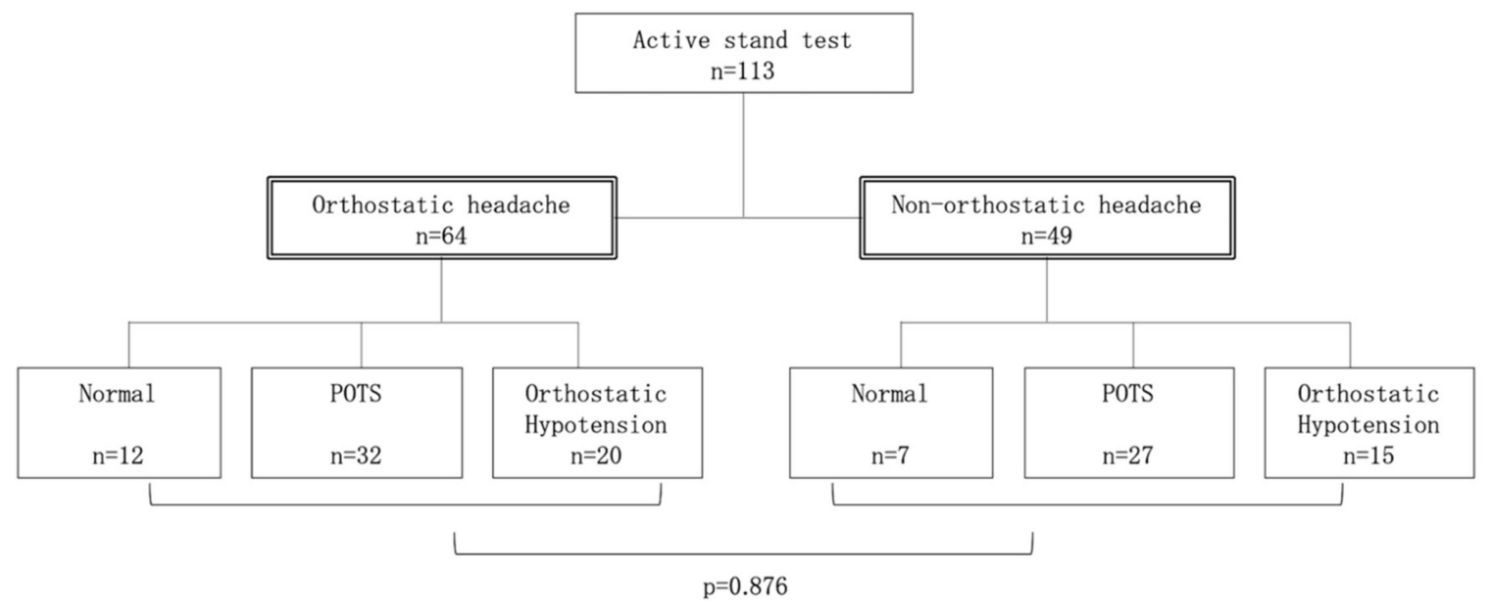

Figure 1. Categorization of participants, orthostatic headache, and autonomic disorders. The inclusion criteria were met by 113 subjects who subsequently underwent the active stand test, including 64 in the orthostatic headache group and 49 in the non-orthostatic headache group. Postural tachycardia syndrome (POTS) was found in 32 subjects (50.0\%) in the orthostatic headache group, and 27 subjects $(55.1 \%)$ in the non-orthostatic headache group. Orthostatic hypotension was found in $20(31.3 \%)$ and $15(30.6 \%)$ subjects in the orthostatic headache and non-orthostatic headache groups, respectively. No statistically significant difference was observed between groups $(p=0.876)$. Values indicate the number of subjects. Statistical analysis was performed using the chi-squared test. $p<0.01$ was considered to indicate statistical significance.

Similarly, the subject characteristics, including age, sex, body constitution, and CTR, did not differ significantly between the groups. Although no significant intergroup difference was observed in the background factors, there were significantly more subjects with a history of migraine in the orthostatic headache group than in the non-orthostatic headache group $(64.1 \%$ vs. $28.6 \% ; p=0.001)$ (Table 1$)$. No significant intergroup differences were observed in the systolic or diastolic blood pressure, heart rate, blood NE level in the supine position at rest, blood pressure recovery time, maximum heart rate, heart rate increment or NE level upon standing. However, a more substantial decrease in cerebral $\mathrm{OxyHb}$ level on the left and right sides after standing was observed in the orthostatic headache group (Left: 6.3 [3.2-9.4] vs. 4.1 [0.8-6.1], $p=0.006$; Right: 5.3 [3.1-8.6] vs. $4.0[0.8-5.9], p=0.007$ ) than in the non-orthostatic headache group. 
Table 1. Participant characteristics and standing test results.

\begin{tabular}{|c|c|c|c|c|}
\hline & & $\begin{array}{c}\text { Orthostatic } \\
\text { Headache } \\
(n=64)\end{array}$ & $\begin{array}{l}\text { Non-Orthostatic } \\
\text { Headache } \\
(n=49)\end{array}$ & $p$-Value \\
\hline \multirow{7}{*}{$\begin{array}{l}\text { Profile and } \\
\text { Background }\end{array}$} & Age (years) & $14.0(13.2-15.1)$ & $14.4(13.2-15.5)$ & 0.491 \\
\hline & Male ratio (\%) & $31 / 64(48.4)$ & $24 / 49(48.9)$ & 0.954 \\
\hline & Height $(\mathrm{cm})$ & $158.9(152.9-164.5)$ & $159.0(152.6-166.2)$ & 0.823 \\
\hline & Weight $(\mathrm{kg})$ & $49.7(40.3-55.3)$ & $49.6(43.7-55.0)$ & 0.956 \\
\hline & BMI & $18.7(16.9-20.9)$ & $19.3(17.7-20.1)$ & 0.876 \\
\hline & CTR $(\%)$ & $42(38-44)$ & $41(38-44)$ & 0.363 \\
\hline & Migraine (\%) & 64.1 & 28.6 & 0.001 * \\
\hline \multirow{4}{*}{ Supine } & $\mathrm{SBP}(\mathrm{mmHg})$ & $108(101-120)$ & $107(99-117)$ & 0.496 \\
\hline & $\mathrm{DBP}(\mathrm{mmHg})$ & $60(51-67)$ & $54(48-63)$ & 0.096 \\
\hline & $\mathrm{HR}(\mathrm{bpm})$ & $66(58-80)$ & $68(64-80)$ & 0.295 \\
\hline & Norepinephrine (pg/mL) & $153(129-225)$ & $160(121-214)$ & 0.842 \\
\hline \multirow{6}{*}{ Standing } & Recovery time (sec) & $20(16-22)$ & $18(15-20)$ & 0.099 \\
\hline & Maximum HR (bpm) & $111(93-125)$ & $111(97-123)$ & 0.768 \\
\hline & HR increase (bpm) & $38(28-51)$ & $38(29-50)$ & 0.871 \\
\hline & Lt cerebral oxyHb decrease $(\mu \mathrm{mol} / \mathrm{L})$ & $6.3(3.2-9.4)$ & $4.1(0.8-6.1)$ & $0.006 *$ \\
\hline & Rt cerebral oxyHb decrease $(\mu \mathrm{mol} / \mathrm{L})$ & $5.3(3.1-8.6)$ & $4.0(0.8-5.9)$ & $0.007 *$ \\
\hline & Norepinephrine (pg/mL) & $352(253-466)$ & $328(252-453)$ & 0.623 \\
\hline
\end{tabular}

The subject characteristics, including age, sex, body constitution, and CTR, did not differ significantly between the groups. Although no significant intergroup difference was observed in the background factors, there were significantly more subjects with a history of migraine in the orthostatic headache group than in the non-orthostatic headache group $(64.1 \%$ vs. $28.6 \% ; p=0.001)$. A more substantial decrease in cerebral OxyHb level on the left and right sides after standing was observed in the orthostatic headache group (Left: 6.3 [3.2-9.4] vs. 4.1 [0.8-6.1], $p=0.006$; Right: 5.3 [3.1-8.6] vs. 4.0 [0.8-5.9], $p=0.007$ ) than in the non-orthostatic headache group. Values are expressed as the median (IQR) or \%. BMI = body mass index, $\mathrm{CTR}=$ cardiothoracic ratio, $\mathrm{SBP}=$ systolic blood pressure, $\mathrm{DBP}=$ diastolic blood pressure, $\mathrm{HR}=$ heart rate, $\mathrm{Rt}=$ right, $\mathrm{Lt}=$ left. Statistical analysis was performed using the Mann-Whitney test. ${ }^{*} p<0.01$ was considered to indicate statistical significance.

Of the 11 somatic symptoms of orthostatic dysregulation (OD) as defined by the Japanese Society of Psychosomatic Pediatrics [8], only headache was experienced most often in the daily life of the subjects in the orthostatic headache group. However, no significant intergroup difference was observed in any other symptoms, including dizziness on standing, fainting in a standing position, nausea on taking a hot bath, palpitations after mild exercise, morning fatigue, face pallor, anorexia, umbilical colic, fatigability, and carsickness (Table 2).

Table 2. Daily physical symptoms.

\begin{tabular}{|c|c|c|c|c|c|c|c|}
\hline & \multicolumn{3}{|c|}{$\begin{array}{l}\text { Orthostatic Headache } \\
\qquad(n=64)\end{array}$} & \multicolumn{3}{|c|}{$\begin{array}{l}\text { Non-Orthostatic Headache } \\
\qquad(n=49)\end{array}$} & \multirow[t]{2}{*}{$p$-Value } \\
\hline & Frequently & Sometimes & Rarely & Frequently & Sometimes & Rarely & \\
\hline 1. Dizziness on standing & 55 & 5 & 4 & 36 & 8 & 5 & 0.156 \\
\hline 2. Fainting in the standing position & 33 & 19 & 12 & 23 & 16 & 10 & 0.843 \\
\hline 3. Nausea on taking a hot bath & 28 & 16 & 20 & 14 & 15 & 20 & 0.227 \\
\hline 4. Palpitations after mild exercise & 31 & 13 & 20 & 16 & 15 & 18 & 0.171 \\
\hline 5. Morning fatigue & 55 & 7 & 2 & 40 & 4 & 5 & 0.310 \\
\hline 6. Face pallor & 17 & 20 & 27 & 21 & 8 & 20 & 0.119 \\
\hline 7. Anorexia & 20 & 13 & 31 & 10 & 10 & 29 & 0.394 \\
\hline 8. Umbilical colic & 28 & 18 & 18 & 17 & 11 & 21 & 0.287 \\
\hline 9. Fatigability & 47 & 11 & 6 & 38 & 8 & 3 & 0.806 \\
\hline 10. Headache & 53 & 8 & 3 & 28 & 9 & 12 & 0.004 * \\
\hline 11. Car sickness & 33 & 15 & 16 & 24 & 15 & 10 & 0.575 \\
\hline
\end{tabular}

Of the 11 somatic symptoms of orthostatic dysregulation (OD) as defined by the Japanese Society of Psychosomatic Pediatrics, headache was experienced most often in the daily life of the subjects in the orthostatic headache group. However, no significant intergroup difference was observed in any of the other symptoms. Values indicate the number of subjects. Statistical analysis was performed using the chi-squared test. * $p<0.01$ was considered to indicate statistical significance. 
The most common site of headache upon standing was the whole head (17 [26.6\%]), followed by the temporal region (16 [25\%]) and the frontal or occipital region (11 [17.2\%]). In 18 of the $64(28 \%)$ children with orthostatic headaches, the headaches induced by the active stand test were pulsatile. No statistical difference was observed in the site of headaches between subjects with and without pulsatile orthostatic headaches $(p=0.214)$ (Table 3A). Neither the proportion of subjects with a high NE level ( $p=0.493)$ nor the prevalence of concomitant migraine $(p=0.441)$ differed between the groups with and without a pulsatile headache (Table 3B). All subjects with an orthostatic headache reported an improvement in their symptom within $5 \mathrm{~min}$ of assuming the supine position after the test.

Table 3. Pulsatility of orthostatic headache.

\begin{tabular}{cccccccc}
\hline & \multicolumn{7}{c}{$(\mathbf{A})$} \\
\hline & Whole head & Frontal & Parietal & Temporal & Posterior & Indeterminate & Total \\
\hline Pulsatility (+) & 5 & 2 & 0 & 8 & 3 & 0 & 18 \\
\hline Pulsatility (-) & 12 & 9 & 4 & 8 & 8 & 5 & 46 \\
\hline Total & 17 & 11 & 4 & 16 & 11 & 5 & 64 \\
\hline \multicolumn{7}{c}{$p=0.214$} \\
\hline
\end{tabular}

(B)

\begin{tabular}{ccccc}
\hline & $\mathrm{NE} \geq 600 \mathrm{pg} / \mathrm{mL}$ & $\mathrm{NE}<600 \mathrm{pg} / \mathrm{mL}$ & Migraine $(+)$ & Migraine $(-)$ \\
\hline Pulsatility (+) & 3 & 15 & 12 & 6 \\
\hline Pulsatility (-) & 5 & 41 & 29 & 17 \\
\hline Total & 8 & 56 & 41 & 23 \\
\hline \multicolumn{2}{c}{$p=0.493$} & \multicolumn{2}{c}{$p=0.441$} \\
\hline
\end{tabular}

The most common site of headache upon standing was the whole head (17 [26.6\%]), followed by the temporal region (16 [25\%]) and the frontal or occipital region (11 [17.2\%]). In 18 of the $64(28 \%)$ children with orthostatic headaches, the headaches induced by the active stand test were pulsatile. No statistical difference was observed in the site of headaches between subjects with and without pulsatile orthostatic headache $(p=0.214)(\mathbf{A})$. Neither the proportion of subjects with a high NE level $(p=0.493)$ nor the prevalence of concomitant migraine $(p=0.441)$ differed between the groups with and without a pulsatile headache $(\mathbf{B})$. Values are number of subjects. Statistical analysis was performed by the Chi-squared tests. $p<0.01$ was considered to indicate statistical significance.

\section{Discussion}

Orthostatic headaches have traditionally been associated with intracranial hypotension. In 2003, Mokri et al. reported that many patients with orthostatic headaches did not, in fact, exhibit features of cerebrospinal fluid leak; moreover, many of them received the diagnosis of POTS following an evaluation of their autonomic nervous system [9]. As a result that the subjects were children, examinations requiring a spinal tap, such as cisternal scintigraphy and magnetic resonance myelography, were not performed to avoid exposing participants unnecessarily to risks associated with these invasive procedures. However, no evidence of intracranial hypotension, such as subdural effusion, dilated epidural venous plexus or diffuse dural enhancement, was observed in the subjects with chronic headaches or episodes of orthostatic headache who had undergone an MRI. In both groups, the median age was almost the same. The gender distribution was slightly skewed toward females. In terms of body constitution, the subjects in both groups were somewhat thin (BMI: 18.7 and 19.3, respectively), with an average $\mathrm{BMI}<22$, which is considered the cutoff value for normal weight. These findings were consistent with the epidemiological evidence of OD in Japan [8]. Previous studies have also shown that BMI tends to be lower in children with POTS than in healthy children [10], a finding also in line with those of the present study. 


\section{1. "Head and/or Neck Pain Attributed to Orthostatic (Postural) Hypotension"-Concept Relevance}

In the present study, 64 of the 113 subjects experienced an orthostatic headache. However, the proportion of normal subjects and those with POTS or OH did not differ significantly between the groups with and without orthostatic headaches. Previously, Deb et al. reported that $87 \%$ of patients with POTS experienced headaches in daily life [11]. Moreover, Khurana et al. reported that among patients with POTS, $58.3 \%$ and $62.5 \%$ developed orthostatic headaches in daily life and during the head-up tilt test, respectively. They also reported that among patients aged $\leq 30$ years, the risk of orthostatic headaches increased the longer they remained in the standing position [12]. In the present study, the 10-min active stand test, which is the standard technique for evaluating autonomic function in children in Japan, induced an orthostatic headache in 32 of the 59 subjects with POTS (54.2\%), which is comparable with previously reported data [12]. It is noteworthy that the previously reported results were reproduced in a laboratory setting. Furthermore, orthostatic headaches occurred in children with POTS without hypotension, such as those with $\mathrm{OH}$; the present study demonstrated that the incidence of orthostatic headaches was higher than expected. Previous studies on vasovagal syncope reported a decrease in the mean blood flow velocity in the middle cerebral artery and an increase in cerebrovascular resistance despite the occurrence of syncope [13,14]. A separate study reported that when presyncope occurred before the onset of deceleration or hypotension, reversible electroencephalographic abnormalities with discrepancies between the right and left signals were observed at the time of syncope aura occurrence [15]. It is plausible that in POTS, in circumstances that cause an abnormal increase in cerebrovascular resistance, failure of tachycardia to compensate for poor blood reflux from the lower extremities or a mild decrease in blood pressure causing a rapid decrease in cerebral blood flow might be experienced as headache, consistent with presyncope symptoms. Our study revealed that the right and left cerebral $\mathrm{OxyHb}$ levels were significantly lower in the orthostatic headache group than in the non-orthostatic headache group. Kim et al., who performed the active stand test in children in the general population without an underlying disease using the same device as in our study, reported that the standing posture did not induce any symptoms in subjects with an $\mathrm{OxyHb}$ decrease $\leq 4 \mu \mathrm{M} / \mathrm{L}$ in contrast to subjects with a decrease $\geq 6.4 \mu \mathrm{M} / \mathrm{L}$, who reported symptoms of OI [16]. These values are comparable with those obtained in our study. Fujita et al. evaluated the cerebral blood flow separately in children with POTS, INOH, and delayed $\mathrm{OH}$ and reported that changes in cerebral blood flow with a discrepancy between the right and left sides preceded changes in blood pressure and heart rate [17-19]. The lack of any difference in the proportion of children with POTS and $\mathrm{OH}$ between the orthostatic headache and non-orthostatic headache groups in our study indicated that orthostatic headaches are a pathological condition that might depend on impaired intracranial homeostasis, which therefore cannot be confirmed by readings of superficial fluctuations in blood pressure and heart rate alone. The concept of "head and/or neck pain attributed to orthostatic (postural) hypotension" included in the ICHD3 should not be restricted by terminology describing superficial changes in the systemic circulation, such as $\mathrm{OH}$ and POTS. Instead, a more inclusive term reflecting the wide variety of possible etiologies should be adopted.

\subsection{Orthostatic Headaches and Norepinephrine}

Thieben et al. reported no association between excretion of urinary sodium $(\mathrm{Na})$, which reflects the circulating blood volume, and increase in noradrenaline level, which reflects sympathetic nervous activity after standing [3]. The authors suggested that failure of the compensatory mechanism for hypovolemia due to peripheral venous pooling or marked leakage from the capillaries in the lower extremities after standing may cause orthostatic headaches.

Meanwhile, the renin-angiotensin-aldosterone system is an important system for maintaining the circulating blood volume. A previous study reported low renin levels in patients with POTS despite hypovolemia [20], drawing attention to insufficient Na retention due to abnormalities in this system as a possible cause of hypovolemia. Although the CTR greatly varies among individuals and does not always accurately reflect the left ventricular volume or circulating blood volume, it is often used as 
a simple indicator in clinical practice. The lack of apparent differences in CTR between the subjects with and without orthostatic headaches in our study indicated that orthostatic headaches might not be caused by changes in the plasma volume, which are reflected in the CTR. Although the normal CTR in children ranged from $41-50 \%$, in the present study, the median CTR was $41 \%$ and $42 \%$ for the orthostatic headache and non-orthostatic headache groups, respectively, suggesting that the subjects in our study with complaints had certain factors which were reflected by low CTR. Low et al. reported that NE levels rose markedly ( $\geq 600 \mathrm{pg} / \mathrm{mL}$ ) upon standing in one-third of his subjects [3,21] and defined hyperadrenergic POTS as an "increase in systolic blood pressure by $10 \mathrm{mmHg}$ or more, and NE level of $600 \mathrm{pg} / \mathrm{mL}$ or higher after standing." Although Zhang et al. reported that the incidence of headache, dizziness, and tremulousness was higher among patients with hyperadrenergic POTS than in those with other types of POTS [22], our study revealed no significant change in the NE level before or after standing between subjects with and without orthostatic headaches. In addition, no association was observed between the pulsatility of orthostatic headaches and blood NE levels upon standing. However, these issues may require further research with a larger number of subjects.

\subsection{Orthostatic Headaches and Migraine}

Ojha et al. reported that the incidence of concomitant migraine in patients with POTS was $41 \%$ in adolescents and $61 \%$ in adult patients [23]. Likewise, Raj et al. reported that $41 \%$ of patients with POTS had a concomitant migraine [24]. Meanwhile, Khurana reported that orthostatic headache in patients with POTS was characterized by moderate-to-severe, migraine-like, pulsatile headaches affecting the whole head or frontal region which were frequently accompanied by photosensitivity, hyperacusis, and nausea. However, the orthostatic headaches tended to be less persistent than migraines in many subjects and were able to be relieved in more than $50 \%$ of the subjects by remaining in the supine position for 2-15 $\mathrm{min}$ [12]. In our study, all subjects with orthostatic headache reported an improvement in their symptoms within 5 min of assuming the supine position, and pulsatility was observed in 18 of the 64 subjects $(28.1 \%)$ in the orthostatic headache group. Furthermore, the subjects in the orthostatic headache group reported headaches more frequently in their daily life than those in the non-orthostatic headache group while the prevalence of migraine was higher in the orthostatic headache group. Given that the orthostatic headache group included 32 subjects with POTS, accounting for half the enrolled subjects, in addition to 20 children with $\mathrm{OH}$, their experience of headache induced by standing might have been misdiagnosed as a migraine by their attending clinicians, who may have relied on headache duration described in the diagnostic criteria for migraine. Miglis reported that migraine with an aura tended to cause more pronounced autonomic dysfunction than migraine without an aura; a higher likelihood of impairment of the sympathetic, rather than the parasympathetic, nervous system; severer sympathetic nervous impairment during the interictal period of a migraine; and increased sympathetic nervous responsiveness during the ictal period, presumably due to adrenoreceptor hypersensitivity [25]. A review article by Gazerani et al. showed a complex pattern of altered autonomic nervous system function associated with migraine, but with an imbalance between the sympathetic and parasympathetic nervous systems [26]. On the other hand, Novak et al. reported that the pathogenesis of POTS appears to involve a hyperadrenergic state and distal neuropathy affecting sympathetic myocardial fibers [27]. Pengo et al. reported that patients with POTS showed neither polysomnographic findings consistent with associated sleep pathology nor objective daytime sleepiness, but subjective daytime sleepiness was associated with increased activation of the parasympathetic nervous system [28]. Gibbons et al. reported cholinergic POTS patients had abnormal proximal sudomotor function and symptoms suggesting gastrointestinal and genitourinary parasympathetic nervous system dysfunction [29]. These may suggest an association between migraine and POTS as an autonomic dysfunction. The present study did not examine the role of the aura, its features, its association with other factors or sympathetic and parasympathetic nervous function; instead, it explored potential differences between typical migraine and orthostatic headaches based on the site of the induced headache, pulsatility, and NE levels upon standing, among other 
considerations. However, no clear trends were found. At present, there is no consensus on treatment strategies for headaches associated with abnormal intracranial homeostasis. Theoretically, common analgesics and triptan are likely to be ineffective [30]. However, the similarity in the clinical features between the orthostatic headaches in our patients and POTS may provide a clue to their treatment.

\subsection{Limitations}

The limitation of this study is that the symptoms, aura, nausea, and photosensitivity associated with orthostatic headache were not assessed, and the association with migraine was not fully investigated. More research is needed on these points in the future.

\section{Conclusions}

Our study found that orthostatic headaches in children were associated with fluctuations in cerebral blood flow and were induced by impaired intracranial homeostasis. In some children, the features of orthostatic headaches were found to be similar to those of migraine. However, in almost all the cases, the headache improved upon assuming the supine position sooner than described in the criteria for migraine diagnosis. Special attention should be paid in clinical practice to this similarity, as the treatment can differ. As orthostatic headaches occur in patients with $\mathrm{OH}$ and POTS, the description, "head and/or neck pain attributed to orthostatic (postural) hypotension" in the ICHD3 should be replaced with a more inclusive, diagnostic term that better defines the condition or differentiates between disparate entities on the basis of head/neck pain attributed to orthostatic hypotension and orthostatic headache associated with impaired intracranial vascular homeostasis. Reconceptualizing orthostatic headaches may help clinicians find more effective treatments and contribute to progress in the research on the disease.

Author Contributions: Data curation, A.K., K.K., T.T., M.T., and N.M.; supervision, Y.K., T.M., N.A., and H.K.; writing-original draft, S.G.; writing-review and editing, G.Y., S.M., Y.I., and S.O. All authors have read and agreed to the published version of the manuscript.

Funding: This study was supported by AMED under Grant Number JP19dk0310099.

Acknowledgments: We would like to show our greatest appreciation to Hidetaka Tanaka, whose enormous support and insightful comments were invaluable during our study.

Conflicts of Interest: The authors declare that they have no conflict of interest.

\section{References}

1. Lateef, T.; Merikangas, K.R.; He, J.; Kalaydjian, A.; Khoromi, S.; Knight, E.; Nelson, K.B. Headache in a national sample of American children: Prevalence and comorbidity. J. Child Neurol. 2009, 24, 536-543. [CrossRef] [PubMed]

2. Low, P.A.; Opfer-Gehrking, T.L.; Textor, S.C.; Benarroch, E.E.; Shen, W.K.; Schondorf, R.; Suarez, G.A.; Rummans, T.A. Postural tachycardia syndrome (POTS). Neurology 1995, 45, S19-S25. [PubMed]

3. Thieben, M.J.; Sandroni, P.; Sletten, D.M.; Benrud-Larson, L.M.; Fealey, R.D.; Vernino, S.; Low, P.A.; Vanda, A.L.; Win-Kuang, S. Postural orthostatic tachycardia syndrome: The Mayo clinic experience. Mayo Clin. Proc. 2007, 82, 308-313. [CrossRef]

4. Headache Classification Committee of the International Headache Society (IHS). The International Classification of Headache Disorders, 3rd edition. Cephalalgia 2018, 38, 1-211. [CrossRef] [PubMed]

5. Mathias, C.J.; Mallipeddi, R.; Bleasdale-Barr, K. Symptoms associated with orthostatic hypotension in pure autonomic failure and multiple system atrophy. J. Neurol. 1999, 246, 893-898. [CrossRef] [PubMed]

6. Cariga, P.; Ahmed, S.; Mathias, C.; Gardner, B. The prevalence and association of neck (coat-hanger) pain and orthostatic (postural) hypotension in human spinal cord injury. Spinal Cord 2002, 40, 77-782. [CrossRef]

7. Tachtsidis, I.; Leung, T.S.; Devoto, L.; Delpy, D.T.; Elwell, C.E. Measurement of frontal lobe functional activation and related systemic effects: A near-infrared spectroscopy investigation. Adv. Exp. Med. Biol. 2008, 614, 397-403. 
8. Tanaka, H.; Fujita, Y.; Takenaka, Y.; Kajiwara, S.; Masutani, S.; Ishizaki, Y.; Matsushima, R.; Shiokawa, H.; Shiota, M.; Ishitani, N.; et al. Japanese clinical guidelines for juvenile orthostatic dysregulation version 1. Pediatr. Int. 2009, 51, 169-179. [CrossRef]

9. Mokri, B.; Low, P.A. Orthostatic headaches without CSF leak in postural tachycardia syndrome. Neurology 2003, 61, 980-982. [CrossRef]

10. Lin, J.; Zhao, H.; Ma, L.; Jiao, F. Body mass index is decreased in children and adolescents with postural tachycardia syndrome. Turk. J. Pediatr. 2019, 61, 52-58. [CrossRef]

11. Deb, A.; Morgenshtern, K.; Culbertson, C.J.; Wang, L.B.; Hohler, A.D. A survey-based analysis of symptoms in patients with postural orthostatic tachycardia syndrome. Proc. Bayl. Univ. Med. Cent. 2015, 28, 157-159. [CrossRef] [PubMed]

12. Khurana, R.K.; Eisenberg, L. Orthostatic and non-orthostatic headache in postural tachycardia syndrome. Cephalalgia 2011, 31, 409-415. [CrossRef] [PubMed]

13. Grubb, B.P.; Gérard, G.; Roush, K.; Temesy-Armos, P.; Montford, P.; Elliott, L.; Hahn, H.; Brewster, P. Cerebral vasoconstriction during head-upright tilt-induced vasovagal syncope. A paradoxic and unexpected response. Circulation 1991, 84, 1157-1164. [CrossRef] [PubMed]

14. Fredman, C.S.; Biermann, K.M.; Patel, V.; Uppstrom, E.L.; Auer, A.I. Transcranial Doppler ultrasonography during head-upright tilt-table testing. Ann. Intern. Med. 1995, 123, 848-849. [CrossRef]

15. Ammirati, F.; Colivicchi, F.; Di Battista, G.; Garelli, F.F.; Pandozi, C.; Santini, M. Variable cerebral dysfunction during tilt induced vasovagal syncope. Pacing Clin. Electrophysiol. 1998, 21, 2420-2425. [CrossRef]

16. Kim, Y.T.; Tanaka, H.; Takaya, R.; Kajiura, M.; Tamai, H.; Arita, M. Quantitative study on cerebral blood volume determined by a near-infrared spectroscopy during postural change in children. Acta Paediatr. 2009, 98, 466-471. [CrossRef]

17. Ishii, W.; Fujita, Y.; Kawaguchi, T.; Kimura, K.; Fukuda, A.; Fuchigami, T.; Morioka, I. Cerebral autoregulation during active standing test in juvenile patients with instantaneous orthostatic hypotension. Adv. Exp. Med. Biol. 2020, 1232, 77-83.

18. Kawaguchi, T.; Fujita, Y.; Ishii, W.; Kimura, K.; Fukuda, A.; Fuchigami, T.; Morioka, I. Cerebral blood oxygenation changes in juvenile patients with delayed orthostatic hypotension during an active standing test. Adv. Exp. Med. Biol. 2020, 1232, 85-90.

19. Kamiyama, Y.; Fujita, Y.; Fuchigami, T.; Kamiyama, H.; Takahashi, S.; Sakatani, K. Asymmetrical changes in cerebral blood oxygenation induced by an active standing test in children with postural tachycardia syndrome. Adv. Exp. Med. Biol. 2014, 812, 271-278.

20. Raj, S.; Biaggioni, I.; Yamhure, P.C.; Black, B.K.; Paranjape, S.Y.; Byrne, D.W.; Robertson, D. Renin-aldosterone paradox and perturbed blood volume regulation underlying postural tachycardia syndrome. Circulation 2005, 111, 1574-1582. [CrossRef]

21. Low, P.A.; Sandroni, P.; Joyner, M.; Shen, W.K. Postural tachycardia syndrome (POTS). J. Cardiovasc. Electrophysiol. 2009, 20, 352-358. [CrossRef] [PubMed]

22. Zhang, Q.; Chen, X.; Li, J.; Du, J. Clinical features of hyperadrenergic postural tachycardia syndrome in children. Pediatr. Int. 2014, 56, 813-816. [CrossRef] [PubMed]

23. Ojha, A.; Chelimsky, T.C.; Chelimsky, G. Comorbidities in pediatric patients with postural orthostatic tachycardia syndrome. J. Pediatr. 2011, 158, 20-23. [CrossRef] [PubMed]

24. Shaw, B.H.; Stiles, L.E.; Bourne, K.; Green, E.A.; Shibao, C.A.; Okamoto, L.E.; Garland, E.M.; Gamboa, A.; Diedrich, A.; Raj, V.; et al. The face of postural tachycardia syndrome-Insights from a large cross-sectional online community-based survey. J. Intern. Med. 2019, 286, 438-448. [CrossRef]

25. Miglis, M.G. Migraine and autonomic dysfunction: Which is the horse and which is the jockey? Curr. Pain Headache Rep. 2018, 22, 19. [CrossRef]

26. Gazerani, P.; Cairns, B.E. Dysautonomia in the pathogenesis of migraine. Expert Rev. Neurother. 2018, 18, 153-165. [CrossRef]

27. Novak, V.; Novak, P.; Opfer-Gehrking, T.L.; O’Brien, P.C.; Low, P.A. Clinical and laboratory indices that enhance the diagnosis of postural tachycardia syndrome. Mayo Clin. Proc. 1998, 73, 1141-1150. [CrossRef]

28. Pengo, M.F.; Higgins, S.; Drakatos, P.; Martin, K.; Gall, N.; Rossi, G.P.; Leschziner, G. Characterisation of sleep disturbances in postural orthostatic tachycardia syndrome: A polysomnography-based study. Sleep Med. 2015, 16, 1457-1461. [CrossRef] 
29. Gibbons, C.H.; Bonyhay, I.; Benson, A.; Wang, N.; Freeman, R. Structural and functional small fiber abnormalities in the neuropathic postural tachycardia syndrome. PLoS ONE 2013, 8, e84716. [CrossRef]

30. Miller, A.J.; Raj, S.R. Pharmacotherapy for postural tachycardia syndrome. Auton. Neurosci. 2018, 215, $28-36$. [CrossRef]

Publisher's Note: MDPI stays neutral with regard to jurisdictional claims in published maps and institutional affiliations.

(C) 2020 by the authors. Licensee MDPI, Basel, Switzerland. This article is an open access article distributed under the terms and conditions of the Creative Commons Attribution (CC BY) license (http://creativecommons.org/licenses/by/4.0/). 